Observat $i$ on of neut ron emi ssi on ani sot ropy by neut $r$ on act $i$ vat $i$ on measur enent in beam i nj ect ed LHD deut er i um pl asmas

\begin{tabular}{|l|l|}
\hline $\begin{array}{l}\text { j our nal or } \\
\text { publ i cat i on } \mathrm{t} \text { i t l e }\end{array}$ & Nucl ear Fusi on \\
\hline vol une & 60 \\
\hline number & 7 \\
\hline page $\mathrm{r}$ ange & 076017 \\
\hline year & $2020-06-15$ \\
\hline URL & ht t p: //hdl . handl e. net /10655/00012852 \\
\hline
\end{tabular}




\title{
Observation of neutron emission anisotropy by neutron activation measurement in beam-injected LHD deuterium plasmas
}

\author{
S Sugiyama ${ }^{1}, \mathbf{T}$ Nishitani $^{2}, \mathbf{H}$ Matsuura $^{1}, \mathbf{M}$ Isobe $^{2,3}$, \\ K Ogawa ${ }^{2,3}$, T Tanaka $^{4}, \mathbf{S}$ Yoshihashi $^{4}$, A Uritani ${ }^{4}$ and \\ M Osakabe Os $^{2,3}$ \\ ${ }^{1}$ Department of Applied Quantum Physics and Nuclear Engineering, Kyushu \\ University, 744 Motooka, Fukuoka 819-0395, Japan \\ 2 National Institute for Fusion Science, National Institutes of Natural Sciences, 322-6 \\ Oroshi-cho, Toki 509-5292. Japan \\ 3 SOKENDAI (The Graduate University for Advanced Studies), 322-6 Oroshi-cho, \\ Toki 509-5292, Japan \\ ${ }^{4}$ Department of Applied Energy, Nagoya University, Furo-cho, Nagoya 464-8603, \\ Japan \\ E-mail: s-sugi@nucl.kyushu-u.ac.jp
}

9 December 2019

\begin{abstract}
Neutron emission anisotropy caused by neutral beam injection was observed by the neutron activation measurement in deuterium plasmas confined in the Large Helical Device. The shot-integrated fast neutron flux was measured by the irradiation of activation foils at two irradiation ends located at different ports. The ratio of the flux at the outside horizontal port to that at the lower port was used as an index of neutron emission anisotropy. The dependence of the ratio on the direction of the neutral beam injection was confirmed by comparing cases of tangential, perpendicular and both tangential and perpendicular neutral beam injections. Neutron emission anisotropy was numerically evaluated assuming these three cases of neutral beam injection. The analysis of neutron emission anisotropy comprises the evaluation of the velocity distribution function of energetic deuterons following guiding-centre orbits, calculation of the double-differential emission spectrum of neutrons produced by the $\mathrm{D}(\mathrm{d}, \mathrm{n})^{3} \mathrm{He}$ reaction and neutron transport calculation. The obtained numerical results are consistent in the dependence of neutron emission anisotropy on the neutralbeam-injection direction with the observed experimental data. The measurement of neutron emission anisotropy can help diagnose the energetic-ion distribution functions, when combined with the measurements of neutron energy spectrum and emission profile and verify the analysis model of the neutron emission spectrum.
\end{abstract}

Keywords: neutron emission anisotropy, neutron activation measurement, neutral beam injection, Large Helical Device

Submitted to: Nucl. Fusion 


\section{Introduction}

In current deuterium plasmas, the information on confined energetic ions can be obtained by the neutron measurement because the $\mathrm{D}(\mathrm{d}, \mathrm{n})^{3} \mathrm{He}$ reaction between energetic and thermal deuterons is dominant in the neutron emission rate. The Large Helical Device (LHD) has several neutron measurement systems [1], including the neutron flux monitor [2] and vertical neutron camera [3]. Using these systems, energetic-particle physics study has been performed in LHD deuterium plasmas [1] such as so-called neutral-beam blip [4], triton burnup experiments [5] and radial transport enhancement of the energetic ion by energetic-ion-driven modes [6].

When fuel-ion velocity distribution functions are anisotropic non-Maxwellian distributions, the emission spectrum of fusion-produced neutrons is anisotropically modified from the Gaussian distribution [7, 8, 9]. For the reactions between anisotropic deuterons, the anisotropy of the neutron emission spectrum is enhanced because of the large anisotropy of the differential cross-section of the $\mathrm{D}(\mathrm{d}, \mathrm{n})^{3} \mathrm{He}$ reaction. Anisotropic non-Maxwellian distribution functions are formed by external heating [10, 11] and excited electromagnetic perturbation [12]. Neutron emission anisotropy can provide further understanding of energetic-particle physics through discussion from the point of view of energetic-ion anisotropy in velocity space and can be an index for the validation of the simulation of energetic-ion behaviour and neutron emission spectrum. Neutral beam (NB) injection easily creates an anisotropic non-Maxwellian tail in the ion velocity distribution function. Therefore, as a first step, it is suitable to compare neutron emission anisotropy between the experiments and analyses. The difference in the neutron emission rate measured by the ${ }^{235} \mathrm{U}$ fission chamber between different detector positions (the top of the LHD centre axis and near the outside port) owing to neutron emission anisotropy caused by tangential NB injection was experimentally observed and explained by numerical analyses [13].

The neutron activation system (NAS) in the LHD measures the number of neutrons emitted during a shot using activation foils [14]. During the same shot, activation foils can be sent to two irradiation ends near the plasma located at the outside and lower ports. The ratio of the induced activity at the outside port to that at the lower port depends on neutron emission anisotropy because of the difference in the range of the neutron emission angle relative to the toroidal axis between the two ports; the ratio can be used to examine the anisotropy. Fusion-produced neutrons are decelerated up to being thermal neutrons by scattering throughout the machine structure, and the sloweddown neutrons drift around the irradiation ends. By choosing the foil material whose reaction with neutrons has a threshold energy, the NAS selectively measures fusionproduced fast neutrons. The irradiation ends of the NAS are closer to the plasma than ${ }^{235} \mathrm{U}$ fission chambers in the LHD. Hence, we can discuss the energetic-ion anisotropy using the NAS more directly than using the neutron flux monitor $\left({ }^{235} \mathrm{U}\right.$ fission chamber). Previously, the dependence of the induced activity ratio of the outside to the lower ports in the NAS on the NB-injection direction and the electron temperature were numerically 
predicted [15]. The prediction showed that the induced activity ratio, when tangential NBs are injected, is larger than that when perpendicular NBs are injected, and this difference is observable in the experiments.

In this paper, we investigated neutron emission anisotropy owing to NB injection in LHD deuterium plasmas by conducting experiments and performing numerical analyses. The dependence of neutron emission anisotropy on the NB-injection direction was observed by comparing three NB-injection cases: the use of tangential, perpendicular and both tangential and perpendicular NB injectors (NBIs). The numerical results are consistent in the dependence on the NB-injection direction with the experimental data. The consistency indicates that the numerical analysis to a certain extent reproduced the energetic-ion behaviour and the neutron emission spectrum.

\section{Experimental setup}

The toroidal magnetic field at the magnetic axis, its direction, and the plasma major radius were $2.75 \mathrm{~T}$, counter clockwise viewed from above and $3.6 \mathrm{~m}$, respectively. In the LHD, three tangential NBIs based on negative-ion sources (NBI\#1, \#2 and \#3) and two perpendicular NBIs based on positive-ion sources (NBI\#4 and \#5) are installed [16], as shown in figure 1 (a). NBI\#1 and \#3 were co-direction injections and NBI\#2 was a counter-direction injection to the toroidal magnetic field. In our experiments, the port-through power and beam energy of NBI\#1-\#5 were approximately $1.4 \mathrm{MW}$ and $177 \mathrm{keV}, 1.2 \mathrm{MW}$ and $150 \mathrm{keV}, 2.1 \mathrm{MW}$ and $169 \mathrm{keV}, 6.0 \mathrm{MW}$ and $55 \mathrm{keV}$ and $8.0 \mathrm{MW}$ and $68 \mathrm{keV}$, respectively.

The NAS measures the shot-integrated neutron yield by exposing the activation foils near the plasma and counting gamma-rays emitted from the irradiated foils [14]. The activation foils are sent by the pneumatic transfer system to two irradiation ends located at the outside (8-O) and lower (2.5-L) ports. Figure 1 shows the positions of the irradiation ends (a) viewed from above and (b) in the poloidal cross-section including the $8-\mathrm{O}$ and 2.5 -L ports. The In foil was used to measure fast neutrons emitted by the $\mathrm{D}(\mathrm{d}, \mathrm{n})^{3}$ He reaction using the ${ }^{115} \operatorname{In}\left(\mathrm{n}, \mathrm{n}^{\prime}\right)^{115 \mathrm{~m}}$ In reaction, which has a threshold energy of $336 \mathrm{keV}$. Thermal neutrons were simultaneously measured using the ${ }^{115} \operatorname{In}(\mathrm{n}, \gamma)^{116 \mathrm{~m}} \operatorname{In}$ reaction. The cross-sections of the ${ }^{115} \operatorname{In}\left(\mathrm{n}, \mathrm{n}^{\prime}\right)^{115 \mathrm{~m}} \operatorname{In}$ and ${ }^{115} \operatorname{In}(\mathrm{n}, \gamma)^{116 \mathrm{~m}} \operatorname{In}$ reactions are shown in figure 2 [17].

Three types of anisotropic deuteron distribution functions were produced using all NBIs (shot number 147429), only two perpendicular NBIs (\#147431), and only three tangential NBIs (\#147433). The In foils were simultaneously sent to the irradiation ends at both the $8-\mathrm{O}$ and 2.5 -L ports. Figure 3 shows the electron cyclotron heating

power $P_{\mathrm{ECH}}$, the port-through beam power $P_{\mathrm{NBI}}$ of the negative-ion-based NBs $(\mathrm{N}-\mathrm{NBs})$ and the positive-ion-based NBs (P-NBs), the central electron density $n_{\mathrm{e} 0}$, the central electron and ion temperatures $T_{\mathrm{e} 0}$ and $T_{\mathrm{i} 0}$, and the total neutron emission rate $S_{\mathrm{n}}$ for the three shots. 


\section{Analysis model}

The steady-state energetic-deuteron distribution function formed by an NB injection was calculated by following guiding-centre orbits of test particles using DELTA5D [18], as follows [9]:

$$
\begin{aligned}
f\left(\mathbf{v}, \frac{r}{a}\right) & =\sum_{j=1}^{N_{\mathrm{t}}} \sum_{i=1}^{N_{\mathrm{p}, j}} \frac{S_{\mathrm{NBI}} \Delta t}{N_{\mathrm{p} 0}} \\
& \times \delta\left(\mathbf{v}-\mathbf{v}_{i, j}\right) \delta\left[\frac{r}{a}-\left(\frac{r}{a}\right)_{i, j}\right] \frac{\mathrm{d}(r / a)}{\mathrm{d} V},
\end{aligned}
$$

where $f$ is the distribution function, $\mathbf{v}$ is the velocity, $r / a$ is the normalized minor radius, $N_{\mathrm{t}}$ is the number of time steps, $N_{\mathrm{p}}$ is the number of the test particles, $S_{\mathrm{NBI}}=P_{\mathrm{NBI}, \mathrm{abs}} / E_{\mathrm{NBI}}, P_{\mathrm{NBI}, \mathrm{abs}}$ is the NB absorption power, $E_{\mathrm{NBI}}$ is the NB-injection energy, $\Delta t$ is the time step interval of the particle orbit calculation, $V$ is the plasma volume, and $\delta$ is the delta function. The subscripts $i$ and $j$ represent the $i$-th particle and the $j$-th time step, respectively. The equilibrium magnetic field was given by the VMEC [19], and the initial distribution of NB-generated deuterons and $P_{\mathrm{NBI}, \text { abs }}$ were determined by the FIT3D [20]. Only beam-thermal Coulomb collision was considered for the velocity change of test particles using the Monte Carlo operator by Boozer and Kuo-Petravic [21]. The guiding-centre orbit of each test particle was followed until the particle either reaches the last closed flux surface or slows down to 1.5 times the ion temperature.

The neutron emission spectrum is defined as follows [7]:

$$
\begin{aligned}
\frac{\mathrm{d}^{2} N}{\mathrm{~d} E \mathrm{~d} \Omega_{\chi}} & =\iiint \mathrm{d} \mathbf{v}_{\mathrm{d}} \mathrm{d} \mathbf{v}_{\mathrm{d}}^{\prime} \mathrm{d} \Omega_{\zeta} \frac{\mathrm{d} \sigma}{\mathrm{d} \Omega_{\zeta}} v_{\mathrm{r}} f_{\mathrm{d}}\left(\mathbf{v}_{\mathrm{d}}, \frac{r}{a}\right) \\
& \times f_{\mathrm{d}}^{\prime}\left(\mathbf{v}_{\mathrm{d}}^{\prime}, \frac{r}{a}\right) \delta\left(E-E_{\mathrm{n}}\right) \delta\left(\Omega_{\chi}-\Omega_{\chi, \mathrm{n}}\right),
\end{aligned}
$$

where $\chi$ is the neutron emission angle relative to the toroidal axis, $\zeta$ is the neutron emission angle in the centre-of-mass system, $\Omega$ is the solid angle, $\mathrm{d} \sigma / \mathrm{d} \Omega_{\zeta}$ is the differential cross-section of the $\mathrm{D}(\mathrm{d}, \mathrm{n})^{3} \mathrm{He}$ reaction in the centre-of-mass system, and $v_{\mathrm{r}}$ is the relative velocity of two reacting deuterons. We considered only the beam-thermal $\mathrm{D}(\mathrm{d}, \mathrm{n})^{3} \mathrm{He}$ reaction; the energetic-deuteron distribution function calculated by (1) and Maxwellian are used for $f_{\mathrm{d}}$ and $f_{\mathrm{d}}^{\prime}$, respectively. $E_{\mathrm{n}}$ is the neutron emission energy calculated using the following formula [22]:

$$
\begin{aligned}
E_{\mathrm{n}} & =\frac{1}{2} m_{\mathrm{n}} v_{\mathrm{c}}^{2}+\frac{m_{3^{3} \mathrm{He}}}{m_{\mathrm{n}}+m_{3} \mathrm{He}}\left(Q+E_{\mathrm{r}}\right) \\
& +v_{\mathrm{c}} \cos \zeta\left[\frac{2 m_{\mathrm{n}} m_{3^{3} \mathrm{He}}}{m_{\mathrm{n}}+m_{3} \mathrm{He}}\left(Q+E_{\mathrm{r}}\right)\right]^{1 / 2},
\end{aligned}
$$

where $m_{\mathrm{n}\left({ }^{3} \mathrm{He}\right)}$ is the neutron $\left({ }^{3} \mathrm{He}\right)$ mass, $v_{\mathrm{c}}$ is the centre-of-mass speed, $Q$ is the reaction $Q$-value of the $\mathrm{D}(\mathrm{d}, \mathrm{n})^{3} \mathrm{He}$ reaction, and $E_{\mathrm{r}}$ is the relative energy of two reacting deuterons. The cross-section of the $\mathrm{D}(\mathrm{d}, \mathrm{n})^{3} \mathrm{He}$ reaction was taken from Drosg and Schwerer [23] and Bosch and Hale [24]. The angular distribution of the differential 
cross-section of the $\mathrm{D}(\mathrm{d}, \mathrm{n})^{3} \mathrm{He}$ reaction in the laboratory system is shown in figure 4 for the deuteron energy $E_{\mathrm{d}}=50,100$ and $200 \mathrm{keV}$; here, $\zeta_{\mathrm{L}}$ is the neutron emission angle in the laboratory system. We evaluated the neutron emission spectrum by calculating (2) based on the Monte Carlo method [9].

The reaction rates of In foils for NAS were calculated by the following formula [13]:

$$
R=\int \mathrm{d} E \sigma_{\operatorname{In}+\mathrm{n}}(E) \phi(E),
$$

where $R$ is the reaction rate of the ${ }^{115} \mathrm{In}+\mathrm{n}$ reaction of the $\mathrm{In}$ foil, $\sigma_{\mathrm{In}+\mathrm{n}}$ is the cross-section of the ${ }^{115} \mathrm{In}+\mathrm{n}$ reaction, and $\phi$ is the neutron energy spectrum at an irradiation end. The neutron energy spectrum $\phi$ was calculated by MCNP-6 [25] with the nuclear data library of FENDL-3.0 [26]. The cross-sections of the ${ }^{115} \operatorname{In}\left(\mathrm{n}, \mathrm{n}^{\prime}\right)^{115 \mathrm{~m}} \operatorname{In}$ and ${ }^{115} \operatorname{In}(\mathrm{n}, \gamma)^{116 \mathrm{~m}} \mathrm{In}$ reactions were obtained from the nuclear data library of the JENDL Dosimetry File 99 [17]. The LHD and the torus hall building were included in the 3-D calculation model by dividing the helical coils and their casing by $6^{\circ}$ toroidal angle pitch and assuming that those components are toroidally symmetric in each $6^{\circ}$-toroidal pitch $[13,27]$. The volume-averaged neutron double-differential spectra calculated by (2) were used for the MCNP-6 calculation as a neutron source, and the shape of a plasma was assumed to be a circular torus with a major radius of $3.6 \mathrm{~m}$ and a minor radius of 0.5 $\mathrm{m}$. To investigate the effect of the energetic-deuteron profile on the reaction rate of In foils, we evaluated the energy spectrum $\phi$ of virgin neutrons that directly enter In foils from the plasma, by considering the neutron double-differential spectra of each position in the plasma and the accurate shape of the vacuum vessel [15, 28].

The port-through NB power, NB energy, electron density, electron and ion temperatures for the calculations were adopted from the experimental data at $4.5 \mathrm{~s}$. We assumed that plasmas are in a steady-state and do not contain impurities (i.e., the effective charge number $Z_{\text {eff }}=1$ ). The ion temperature in the shot \#147433 could not be obtained; it was supposed to be same as that in the shot \#147429 for the calculation. The density and temperature profiles were measured as a function of the major radius $R_{\text {maj }}$ along the equatorial plane in a poloidal cross-section in the experiment and could be converted to those as a function of the normalized minor radius $r / a$ using the relation between $R_{\text {maj }}$ and $r$ /a obtained from the VMEC calculation. The profiles of the electron density, electron and ion temperatures for the calculations were determined by quartic, cubic and quadratic polynomials with respect to the normalized toroidal flux function

$\psi=(r / a)^{2}$ generated from the experimental data at $4.5 \mathrm{~s}$ with the least squares method, respectively. The experimentally observed and fitted profiles of (a) the electron density and (b) the electron and ion temperatures in shot \#147429 are shown in figure 5.

\section{Results and discussion}

All neutron emission angles relative to the toroidal axis $\chi$ can enter the irradiation end at the $8-\mathrm{O}$ port, while neutrons that enter at the 2.5 - $\mathrm{L}$ port are limited to the intermediate emission angle, geometrically. We define the parameters that estimate neutron emission 
anisotropy as $\eta=R_{\mathrm{O}} / R_{\mathrm{L}}$ and $\eta^{\prime}=R_{\mathrm{O}}^{\prime} / R_{\mathrm{L}}^{\prime}$. Here, $R_{\mathrm{O}(\mathrm{L})}$ and $R_{\mathrm{O}(\mathrm{L})}^{\prime}$ are the reaction rates of the ${ }^{115} \operatorname{In}\left(\mathrm{n}, \mathrm{n}^{\prime}\right)^{115 \mathrm{~m}} \mathrm{In}$ and ${ }^{115} \operatorname{In}(\mathrm{n}, \gamma)^{116 \mathrm{~m}} \mathrm{In}$ reactions of $\mathrm{In}$ foil sent to the irradiation ends at the 8 -O (2.5-L) port, respectively. The parameters $\eta$ and $\eta^{\prime}$ are determined by the difference in the neutron emission angle at which the neutrons are allowed to enter the irradiation end and by the geometric relationship of the neutron emission profile to the irradiation end between the 8-O and 2.5-L ports.

The reaction rates $R_{\mathrm{O}}, R_{\mathrm{L}}, R_{\mathrm{O}}^{\prime}$ and $R_{\mathrm{L}}^{\prime}$ and the parameters $\eta$ and $\eta^{\prime}$ obtained from the experiments are shown in table 1 . The difference in $\eta$ between the cases of perpendicular NB injection (\#147431) and tangential injection (\#147433) is clearly observed. The parameter $\eta$ for all NB injections (\#147429) is almost the same as that in the case of tangential injection because the reaction rate of the $\mathrm{D}(\mathrm{d}, \mathrm{n})^{3} \mathrm{He}$ reaction mainly consists of the reactions of deuterons generated by tangential NB injection as can be seen by the comparison of the neutron emission rates $S_{\mathrm{n}}$ in the shots \#147431 and \#147433, which are shown in figure 3. However, the significant difference in $\eta^{\prime}$ between three cases is not observed. This occurs because thermal neutrons mostly contribute to the ${ }^{115} \operatorname{In}(\mathrm{n}, \gamma)^{116 \mathrm{~m}} \mathrm{In}$ reaction and they lose neutron emission anisotropy by multiple scattering throughout the machine structure. Therefore, $\eta^{\prime}$ is determined by the difference in materials and their configuration around the irradiation end between the 8-O port and the 2.5-L port. A comparison between the dependences of $\eta$ and $\eta^{\prime}$ on the NB-injection direction indicates that neutron emission anisotropy certainly affects the value of $\eta$ in addition to the difference in position of the ports.

The volume-averaged energetic-deuteron distribution functions calculated from (1) following the particle guiding-centre orbits are shown in figure 6 for the cases of (a) all NB injection (\#147429), (b) perpendicular injection (\#147431) and (c) tangential injection (\#147433). Here, $v_{\|}$and $v_{\perp}$ are the velocity components that are parallel and perpendicular to the magnetic field, respectively, and $v_{180}$ represents the $180-\mathrm{keV}$ deuteron speed. The black dots show the NB injection velocity. The different types of anisotropy of the energetic-deuteron distribution functions are produced by varying the NB-injection pattern. The shown distribution functions consist of anisotropic slowingdown distributions formed in the direction of each NBI. Six peaks along $v_{\perp}$ near $v_{\|}=0$ are observed for all and perpendicular injections, which stem from the components of $E_{\mathrm{NBI}} / 2$ and $E_{\mathrm{NBI}} / 3$ that are included in the positive-ion-based NBIs (NBI\#4 and NBI\#5). For the case of tangential NB injection, the fraction of energetic-deuteron population near the ion pitch $\cos \left(v_{\|} / v\right)=0$ is low even by taking into account the lack of perpendicular NB injection. This occurs because energetic ions with the pitch around zero are in the orbit-loss region in velocity space.

The volume-averaged double-differential neutron emission spectra are shown in figure 7 for the cases of (a) all NB injection, (b) perpendicular injection and (c) tangential injection. These spectra are calculated using (2) and the energetic-deuteron distribution functions shown in figure 6 . Here, $\chi$ is the neutron emission angle relative to the toroidal axis, and the direction of $\chi=0^{\circ}$ is counter clockwise when viewed from above, i.e., the same direction as NBI\#1 and \#3. As seen from (3), the maximum possible neutron 
energy is observed in the same direction as the direction of motion of reacting energetic deuteron, while the minimum energy is observed in the opposite direction. For tangential NB injection, the reactions of energetic deuterons owing to the co-direction injectors (NBI\#1 and \#3) form a higher energy component than $2.45 \mathrm{MeV}$ at $\chi=0^{\circ}$ and the lower energy component at $\chi=180^{\circ}$ in the neutron emission spectra. Owing to the counter-direction injector (NBI\#2), the higher and lower energy components are created at $\chi=180^{\circ}$ and $0^{\circ}$, respectively. Therefore, for tangential injection, two peaks appear at $\chi=0^{\circ}$ and $180^{\circ}$, and an intermediate energy component is observed at $\chi=90^{\circ}$ in the spectra shown in figure 7 (c). For perpendicular injection, neutrons in the entire energy range can be observed at $\chi=90^{\circ}$, and the energy spectra at $\chi=0^{\circ}$ and $180^{\circ}$ have only an intermediate energy component because of the cyclotron motion of the perpendicularly moving energetic deuterons. The neutron spectrum at $\chi=90^{\circ}$ shows two peaks owing to perpendicular injectors (NB\#4 and \#5) as those in figure 7 (b). The shape of the neutron spectra in figure 7 is understood as the superposition of cases of tangential and perpendicular injection.

We define neutron emission anisotropy as $2\left(\mathrm{~d} N / \mathrm{d} \Omega_{\chi}\right)_{\text {normalized }}-1$, i.e., the relative difference in the angular distributions of neutron spectra between anisotropic and isotropic emissions. Here, $\left(\mathrm{d} N / \mathrm{d} \Omega_{\chi}\right)_{\text {normalized }}$ is the integral of the neutron emission spectra in figure 7 with respect to the neutron energy and normalized by the neutron emission rate. The neutron emission anisotropy is shown in figure 8 for all (\#147429), perpendicular (\#147431) and tangential (\#147433) NB-injection cases. The emission rate along the neutron emission angles in the centre-of-mass system $\zeta=0^{\circ}$ and $180^{\circ}$ is higher than that along $\zeta=90^{\circ}$ owing to the angular distribution of the differential crosssection of the $\mathrm{D}(\mathrm{d}, \mathrm{n})^{3} \mathrm{He}$ reaction. The centre-of-mass velocity enhances the forward emission rate in the laboratory system; the emission rate along the neutron emission angle in the laboratory system $\zeta_{\mathrm{L}}$ of $0^{\circ}$ is higher than that along $\zeta_{\mathrm{L}}=180^{\circ}$. For tangential NB injection, the NB-generated deuterons move almost along the magnetic field line; hence, $\chi$ is regarded as $\zeta_{\mathrm{L}}$. The neutron emission anisotropy for the case of tangential injection (\#147433) is similar to the angular distribution of differential cross-section in the laboratory system shown in figure 4. For neutrons emitted by the reactions of perpendicularly moving energetic deuterons, all $\zeta_{\mathrm{L}}$ are allowed to be $\chi=90^{\circ}$, whereas $\chi=0^{\circ}$ and $180^{\circ}$ originate from only $\zeta_{\mathrm{L}}$ of $90^{\circ}$. Accordingly, the emission rate along $\chi=90^{\circ}$ increases and those along $\chi=0^{\circ}$ and $180^{\circ}$ decrease for the perpendicular NB-injection case (\#147431). Again, the case of all NB injection is a superposition of the tangential and perpendicular injection cases. Neutrons emitted in $\chi=0^{\circ}$ and $180^{\circ}$ directions cannot geometrically enter the In foil at the 2.5-L port, whereas neutrons emitted in all directions $\chi$ can enter the foil at the 8-O port. Therefore, as the emission fractions at $\chi=0^{\circ}$ and $180^{\circ}$ increase, the parameter $\eta$ becomes large, and vice versa. Thus, $\eta$ for the case of tangential NB injection is expected to be larger than that for the case of perpendicular injection.

We compare the parameters $\eta$ and $\eta^{\prime}$ calculated with those obtained from the experiments in table 2. Here, analysis (A) is the neutron transport calculation using 
the MCNP-6, assuming the neutron source is a uniform circular torus with energy and angular distributions shown in figure 7, and analysis (B) means the calculation for only virgin neutrons entering In foils directly from the plasma, considering the energeticdeuteron profile. The difference in $\eta$ between analyses (A) and (B) originates from the slowing-down energy component in neutron flux of more than $336 \mathrm{keV}$ and the neutron emission profile. When the tangential NBs are injected, the energetic deuterons concentrate in the plasma core [29]; the uniform-torus neutron source is a sufficiently good approximation for the calculation of $\eta$. Hence, for the tangential NB-injection case (\#147433), $\eta$ obtained from analysis (A) has a smaller value than that obtained from analysis (B) because of a decrease in the anisotropy by scattered neutrons throughout the machine structure. Meanwhile, the energetic-deuteron profile has a peak at a lower part than at the equatorial plane owing to helically trapped deuterons in the poloidal plane including the 2.5-L port when the perpendicular NBs are injected [29]. This concentration of energetic deuterons decreases the value of $\eta$ because the main region of neutron emission comes closer to the irradiation end at the 2.5-L port than for the case of the uniform-torus neutron emission. Therefore, $\eta$ obtained from analysis (A) is larger than that from analysis (B) for the case of perpendicular NB injection (\#147431). Considering these differences between analysis (A) and (B), the value $\eta$ estimated from analysis (B) includes more accurate information on energetic deuterons. The dependence of neutron emission anisotropy on the NB-injection direction can be understood not by the absolute value of $\eta$ but by the magnitude relationship of $\eta$ between the shots. The normalized values of $\eta$ for the all NB injection case (\#147429) by $\eta$ for the perpendicular injection case (\#147431) obtained by the experiments and analysis (B) are 1.68 and 1.69, and those for the tangential NB injection case (\#147433) are 1.67 and 1.85, respectively. The numerical results are consistent in the dependence of neutron emission anisotropy on the NB-injection direction with the observed experimental data.

In table 2 , the dependence of $\eta^{\prime}$ on the NB-injection direction and the absolute values of $\eta$ and $\eta^{\prime}$ are different between the experiments and numerical analyses. The inconsistency in the dependence and absolute value of $\eta^{\prime}$ is due to the lack of modelling of several materials that contribute to the slowing down of neutrons in the 3-D calculation model of the LHD for the MCNP-6; this characteristic does not affect the difference in the absolute values of $\eta$, which primarily originates from the modelling of the wall near the 2.5-L port. Because the size of the 2.5-L port determines the plasma region viewed from the In foil at this port, the modelling of the size and shape of the port considerably influences the absolute value of $\eta$; however, the analysis model does not accurately include the port shape. To use parameter $\eta$ for the validation of the simulation on energetic-ion behaviour as an index, we need to be able to discuss its absolute value, i.e., we need to improve the analysis model of the shape of the LHD wall.

For intense NB injection, the beam-beam collision can be important for the formation of the energetic-deuteron distribution function [30] especially the centre of plasma. In this case, the nonlinear Coulomb collision effect is important for the accurate simulation on energetic-ion behaviour and changes the neutron emission anisotropy. 
This effect on neutron emission anisotropy can be confirmed by investigating the NBpower dependence of $\eta$ by varying the number of NBIs. Because radio-frequency waves in the ion cyclotron range of frequencies (ICRF) can produce $\mathrm{MeV}$-order ions [31] by accelerating ions perpendicular to magnetic field lines, the different characteristics of $\eta$ from the case of NB injection must be observed in ICRF-heated plasmas. The time evolution of the plasma density and temperature and the existence of the electric field also affect the energetic-deuteron distribution function. We can understand the energetic-ion velocity distribution functions formed according to the abovementioned effects and confinement property from the parameter $\eta$ by simultaneously measuring the neutron energy spectrum and emission profile.

\section{Conclusion}

We conducted experiments to investigate neutron emission anisotropy caused by NB injection in LHD deuterium plasmas by the neutron activation measurement. Neutron emission anisotropy was estimated by introducing the ratio of the reaction rate of the ${ }^{115} \mathrm{In}+\mathrm{n}$ reaction at the irradiation end at the $8-\mathrm{O}$ port to that at the 2.5 - $\mathrm{L}$ port of the NAS. The dependence of the ratio $\eta$ for the ${ }^{115} \operatorname{In}\left(\mathrm{n}, \mathrm{n}^{\prime}\right)^{115 \mathrm{~m}} \mathrm{In}$ reaction on NBinjection direction was observed, while the ratio $\eta^{\prime}$ for the ${ }^{115} \operatorname{In}(\mathrm{n}, \gamma)^{116 \mathrm{~m}} \mathrm{In}$ reaction was independent. In addition, we performed numerical analyses of the experiments. The obtained numerical results agreed with the experimental data for the dependence of $\eta$. This consistency indicates that our analysis model to a certain extent reproduces the velocity distribution functions of energetic deuterons generated by NB injection and the emission spectrum of fusion-produced neutrons.

The absolute values of $\eta$ and $\eta^{\prime}$ and the dependence of $\eta^{\prime}$ on the NB-injection direction obtained from the numerical analyses were different from those experimentally observed. The inconsistency in the absolute value of $\eta$ is mainly caused by the modelling of the shape of the LHD wall, and the dependence and absolute value of $\eta^{\prime}$ stem from the lack of materials that contribute to the deceleration of neutrons in the 3-D calculation model for MCNP-6. To discuss energetic-particle physics and the validity of simulation of energetic-ion behaviour using $\eta$, it is necessary to improve our geometric model of the LHD.

Other than NB injection, neutron emission anisotropy as a result of anisotropic distribution functions of energetic ions can be caused by various phenomena such as ICRF heating and Alfvén eigenmodes [32]. The neutron activation measurement at two irradiation ends can be expected to provide experimental understanding of the energeticion velocity distribution functions by combining with the measurements of the neutron energy spectrum and emission profile. 


\section{Acknowledgments}

The authors would like to thank LHD Experiment Group for their contributions to the experiments on neutron emission anisotropy. This work was supported by JSPS, Grant-in-Aid for JSPS Research Fellow, 18J12685.

\section{References}

[1] Isobe M, Ogawa K, Nishitani T, Pu N, Kawase H, Seki R, Nuga H, Takada E, Murakami S, Suzuki Y, Yokoyama M, Osakabe M and LHD Experiment Group 2018 Nucl. Fusion 58082004

[2] Isobe M, Ogawa K, Miyake H, Hayashi H, Kobuchi T, Nakano Y, Watanabe K, Uritani A, Misawa T, Nishitani T, Tomitaka M, Kumagai T, Mashiyama Y, Ito D, Kono S, Yamauchi M and Takeiri Y 2014 Rev. Sci. Instrum 85 11E114

[3] Ogawa K, Isobe M, Nishitani T and Kobuchi T 2018 Rev. Sci. Instrum 89113509

[4] Nishitani T, Ogawa K, Pu N, Kawase H, Murakami S, Isobe M, Osakabe M and the LHD Experimental Group 2018 Plasma Fusion Res. 133402024

[5] Ogawa K, Isobe M, Nishitani T, Murakami S, Seki R, Nakata M, Takada E, Kawase H, Pu N and LHD Experiment Group 2018 Nucl. Fusion 58034002

[6] Ogawa K, Isobe M, Kawase H, Nishitani T, Seki R, Osakabe M and LHD Experiment Group 2018 Nucl. Fusion 58044001

[7] Matsuura H and Nakao Y 2010 J. Plasma Fusion Res. SERIES 948

[8] Goncharov P R 2015 Nucl. Fusion 55063012

[9] Sugiyama S, Matsuura H and Uchiyama D 2017 Phys. Plasmas 24092517

[10] Cordey J G and Houghton M J 1973 Nucl. Fusion 13215

[11] Stix T H 1975 Nucl. Fusion 15737

[12] Sugiyama S, Matsuura H and Ogawa K 2018 Plasma Phys. Control. Fusion 60105003

[13] Nishitani T, Matsuura H, Pu N, Ogawa K, Kawase H, Isobe M and the LHD Experiment Group 2019 IEEE Trans. Plasma Sci. 4712

[14] Pu N, Nishitani T, Isobe M, Ogawa K, Kawase H, Tanaka T, Li S Y, Yoshihashi S and Uritani A 2017 Rev. Sci. Instrum. 88113302

[15] Sugiyama S, Matsuura H, Goto T, Nishitani T, Isobe M and Ogawa K 2019 Plasma Fusion Res. 143403123

[16] Osakabe M, Isobe M, Tanaka M, Motojima G, Tsumori K, Yokoyama M, Morisaki T, Takeiri Y and LHD Experiment Group 2018 IEEE Trans. Plasma Sci. 462324

[17] Kobayashi K, Iguchi T, Iwasaki S, Aoyama T, Shimakawa S, Ikeda Y, Odano N, Sakurai K, Shibata K, Nakagawa T and Nakazawa M 2002 JENDL Dosimetry File 99 (JENDL/D-99) JAERI 1344 Japan Atomic Energy Research Institute Tokai

[18] Spong D A 2011 Phys. Plasmas 18056109

[19] Hirshman S P and Whitson J C 1983 Phys. Fluids 263553

[20] Murakami S, Nakajima N and Okamoto M 1995 Trans. Fusion Technol. 27256

[21] Boozer A H and Kuo-Petravic G 1981 Phys. Fluids 24851

[22] Brysk H 1973 Plasma Phys. 15611

[23] Drosg M and Schwerer O 1987 Production of monoenergetic neutrons between 0.1 and $23 \mathrm{MeV}$ : neutron energies and cross-sections Handbook on Nuclear Activation Data STI/DOC/10/273 IAEA Vienna

[24] Bosch H -S and Hale G M 1992 Nucl. Fusion 32611

[25] Goorley T 2014 MCNP6.1.1-Beta Release Notes LA-UR-14-24680 Los Alamos National Laboratory Los Alamos

[26] Forrest R A, Capote R, Otsuka N, Kawano T, Koning A J, Kunieda S, Sublet J-Ch and Watanabe 
Y 2012 FENDL-3 Library - Summary documentation IAEA Report INDC(NDS)-628 IAEA Vienna

[27] Nishitani T, Ogawa K and Isobe M 2017 Fusion Eng. Des. 1231020

[28] Sugiyama S, Matsuura H and Goto T 2016 Plasma Fusion Res. 112403049

[29] Ogawa K, Isobe M, Kawase H, Nishitani T, Seki R, Osakabe M and LHD Experiment Group 2018 Plasma Phys. Control. Fusion 60044005

[30] Nuga H, Seki R, Kamio S, Ogawa K, Isobe M, Osakabe M, Yokoyama M and LHD Experiment Group 2019 Nucl. Fusion 59016007

[31] Mutoh T, Kumazawa R, Seki T, Saito K, Kasahara H, Nakamura Y, Masuzaki S, Kubo S, Takeiri Y, Shimozuma T, Yoshimura Y, Igami H, Watanabe T, Ogawa H, Miyazawa J, Shoji M, Ashikawa N, Nishimura K, Osakabe M, Tsumori K, Ikeda K, Nagaoka K, Oka Y, Chikaraishi H, Funaba H, Morita S, Goto M, Inagaki S, Narihara K, Tokuzawa T, Sakamoto R, Morisaki T, Peterson B, Tanaka K, Nakanishi H, Nishiura M, Ozaki T, Shimpo F, Nomura G, Takahashi C, Yokota M, Zhao Y, Kwak J, Murakami S, Okada H, Yamada H, Kawahata K, Ohyabu N, Kaneko O, Ida K, Nagayama Y, Watanabe K, Noda N, Komori A, Sudo S and Motojima O 2007 Nucl. Fusion $\mathbf{4 7} 1250$

[32] Sugiyama S and Matsuura H 2019 Fusion Eng. Des. 146320 
Table 1. Reaction rates of In foils and the parameters for neutron emission anisotropy $\eta=R_{\mathrm{O}} / R_{\mathrm{L}}$ and $\eta^{\prime}=R_{\mathrm{O}}^{\prime} / R_{\mathrm{L}}^{\prime} . \quad R_{\mathrm{O}(\mathrm{L})}$ and $R_{\mathrm{O}(\mathrm{L})}^{\prime}$ are the reaction rates of the ${ }^{115} \operatorname{In}\left(\mathrm{n}, \mathrm{n}^{\prime}\right)^{115 \mathrm{~m}} \operatorname{In}$ and ${ }^{115} \operatorname{In}(\mathrm{n}, \gamma)^{116 \mathrm{~m}} \mathrm{In}$ reactions at the irradiation end of the 8-O (2.5-L) port, respectively.

\begin{tabular}{|c|c|c|c|c|c|c|}
\hline \multirow[b]{2}{*}{ Shot No. } & \multicolumn{2}{|c|}{ Reaction rate $\left[\mathrm{s}^{-1}\right]$} & \multirow[b]{2}{*}{$\eta$} & \multicolumn{2}{|c|}{ Reaction rate $\left[\mathrm{s}^{-1}\right]$} & \multirow[b]{2}{*}{$\eta^{\prime}$} \\
\hline & $R_{\mathrm{O}}$ & $R_{\mathrm{L}}$ & & $R_{\mathrm{O}}^{\prime}$ & $R_{\mathrm{L}}^{\prime}$ & \\
\hline 147429 & $1.61 \times 10^{6}$ & $2.87 \times 10^{5}$ & $5.63 \pm 0.15$ & $3.74 \times 10^{7}$ & $2.15 \times 10^{7}$ & $1.74 \pm 0.05$ \\
\hline 147431 & $2.63 \times 10^{5}$ & $7.85 \times 10^{4}$ & $3.35 \pm 0.18$ & $8.36 \times 10^{6}$ & $4.80 \times 10^{6}$ & $1.74 \pm 0.02$ \\
\hline 147433 & $1.15 \times 10^{6}$ & $2.06 \times 10^{5}$ & $5.58 \pm 0.33$ & $2.75 \times 10^{7}$ & $1.49 \times 10^{7}$ & $1.84 \pm 0.05$ \\
\hline
\end{tabular}

Table 2. Parameters for neutron emission anisotropy obtained from experiments and numerical analyses.

\begin{tabular}{ccccccc}
\hline & \multicolumn{3}{c}{$\eta$} & & \multicolumn{2}{c}{$\eta^{\prime}$} \\
\cline { 2 - 3 } \cline { 6 - 7 } Shot No. & Experiment & Analysis (A) & Analysis (B) & & Experiment & Analysis (A) \\
\hline 147429 & 5.63 & 3.780 & 3.521 & & 1.74 & 0.3269 \\
147431 & 3.35 & 2.904 & 2.078 & & 1.74 & 0.2701 \\
147433 & 5.58 & 3.565 & 3.838 & & 1.84 & 0.3048 \\
\hline
\end{tabular}

${ }^{a}$ Neutron transport calculation using MCNP-6. ${ }^{\mathrm{b}}$ Calculation for only virgin neutrons that directly enter In foils from the plasma. 


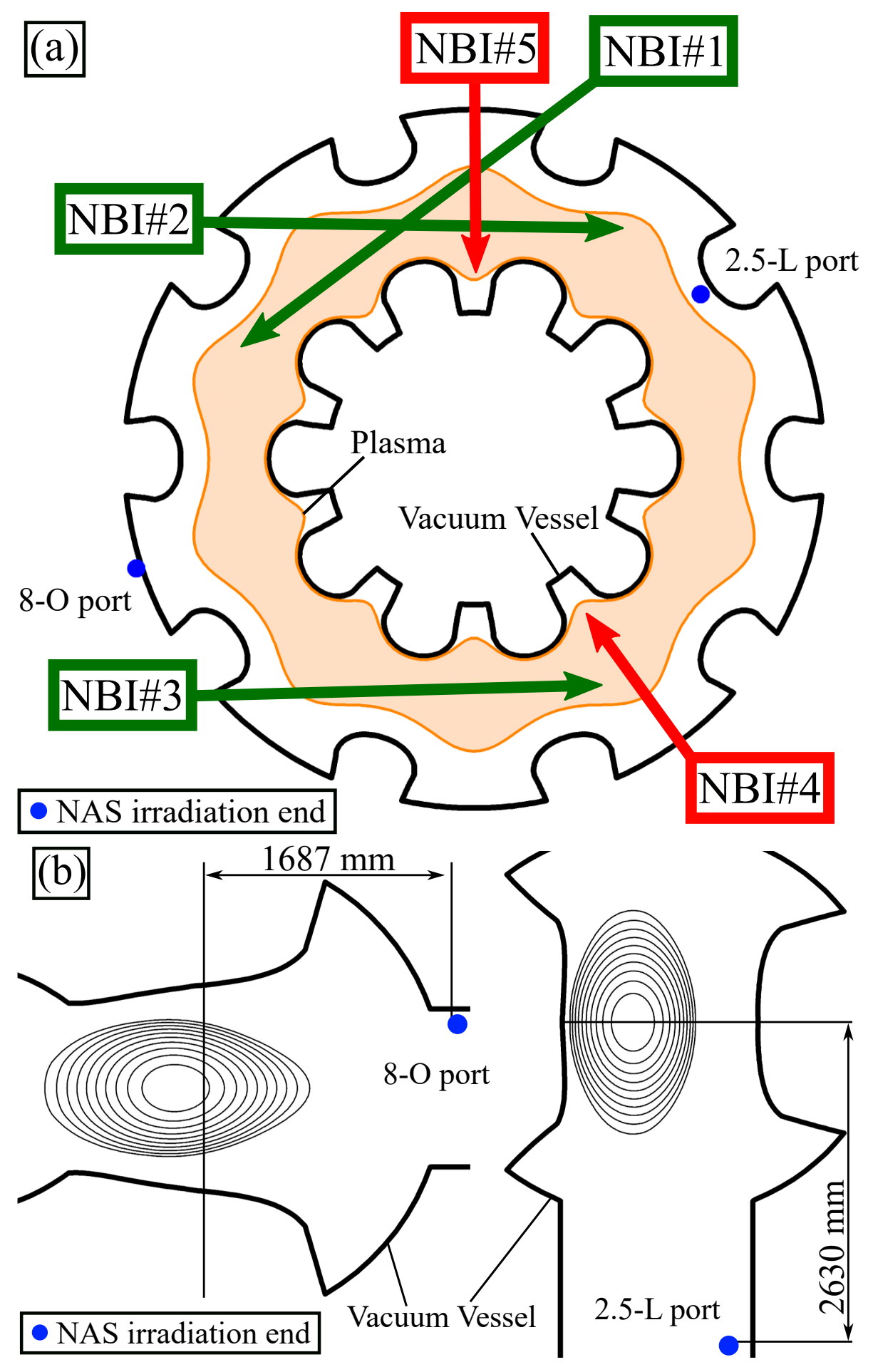

Figure 1. Schematics of (a) NBIs and irradiation ends of NAS on LHD viewed from above and (b) irradiation ends in poloidal planes including 8-O and 2.5-L ports. 


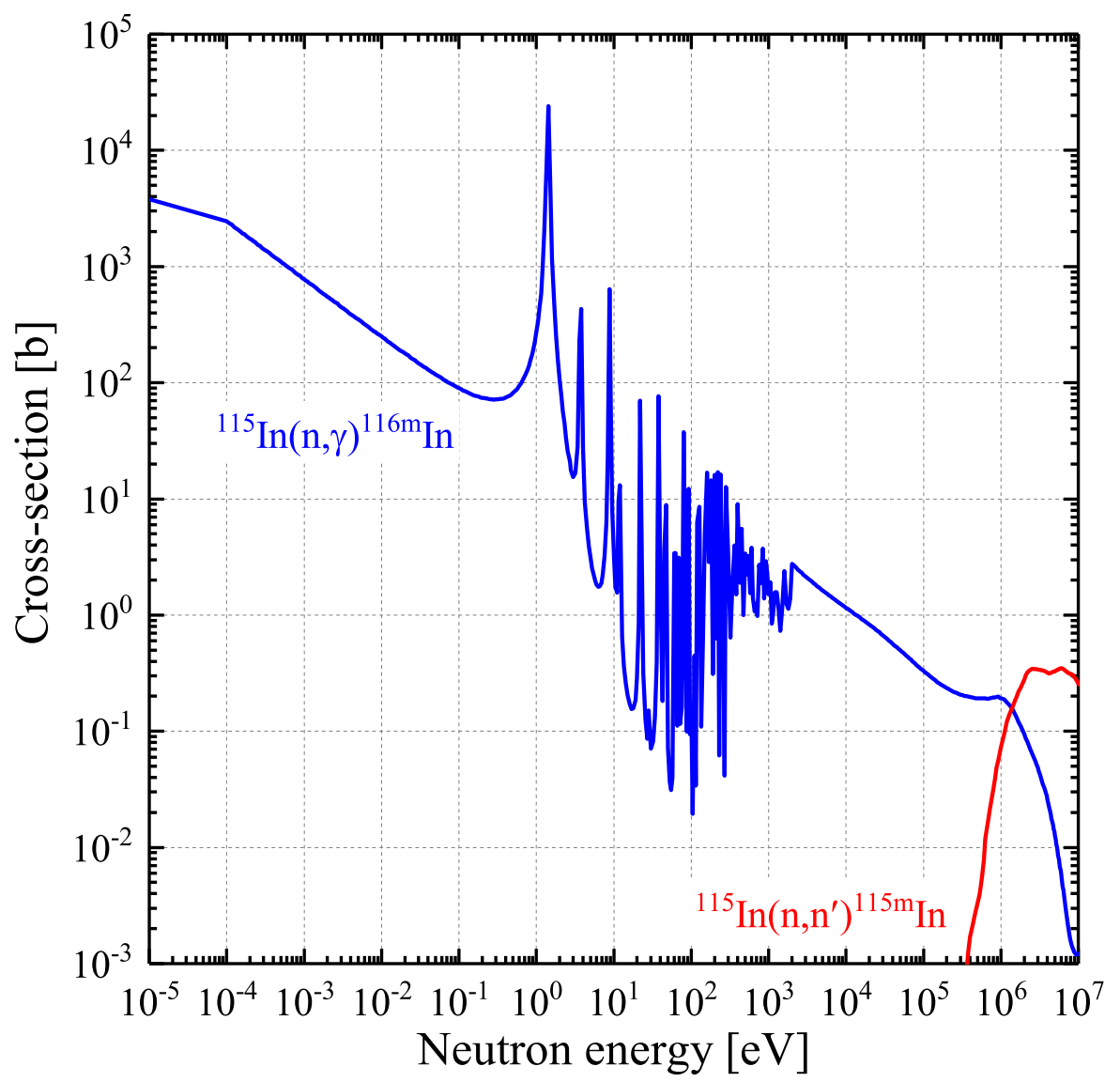

Figure 2. Cross-sections of ${ }^{115} \operatorname{In}\left(\mathrm{n}, \mathrm{n}^{\prime}\right){ }^{115 \mathrm{~m}} \operatorname{In}$ and ${ }^{115} \operatorname{In}(\mathrm{n}, \gamma){ }^{116 \mathrm{~m}} \mathrm{In}$ reactions [17]. 


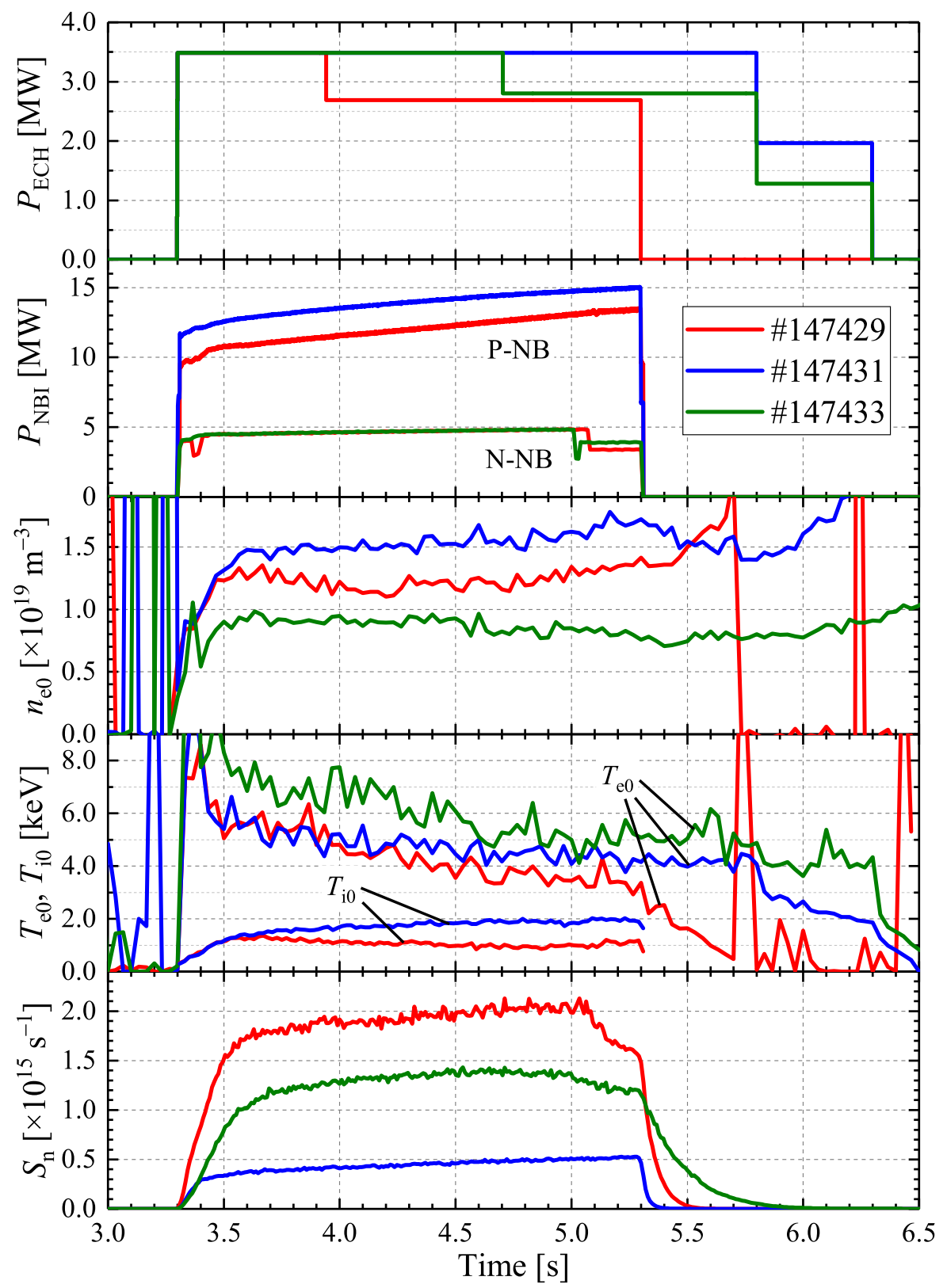

Figure 3. Time evolutions of electron cyclotron heating power $P_{\mathrm{ECH}}$, port-through NB power $P_{\mathrm{NBI}}$ of negative-ion-based NBs (N-NBs) and positive-ion-based NBs (P$\mathrm{NBs}$ ), central electron density $n_{\mathrm{e} 0}$, central electron and ion temperatures $T_{\mathrm{e} 0}$ and $T_{\mathrm{i} 0}$, and neutron emission rate $S_{\mathrm{n}}$ for the three shots \#147429, \#147431 and \#147433. 


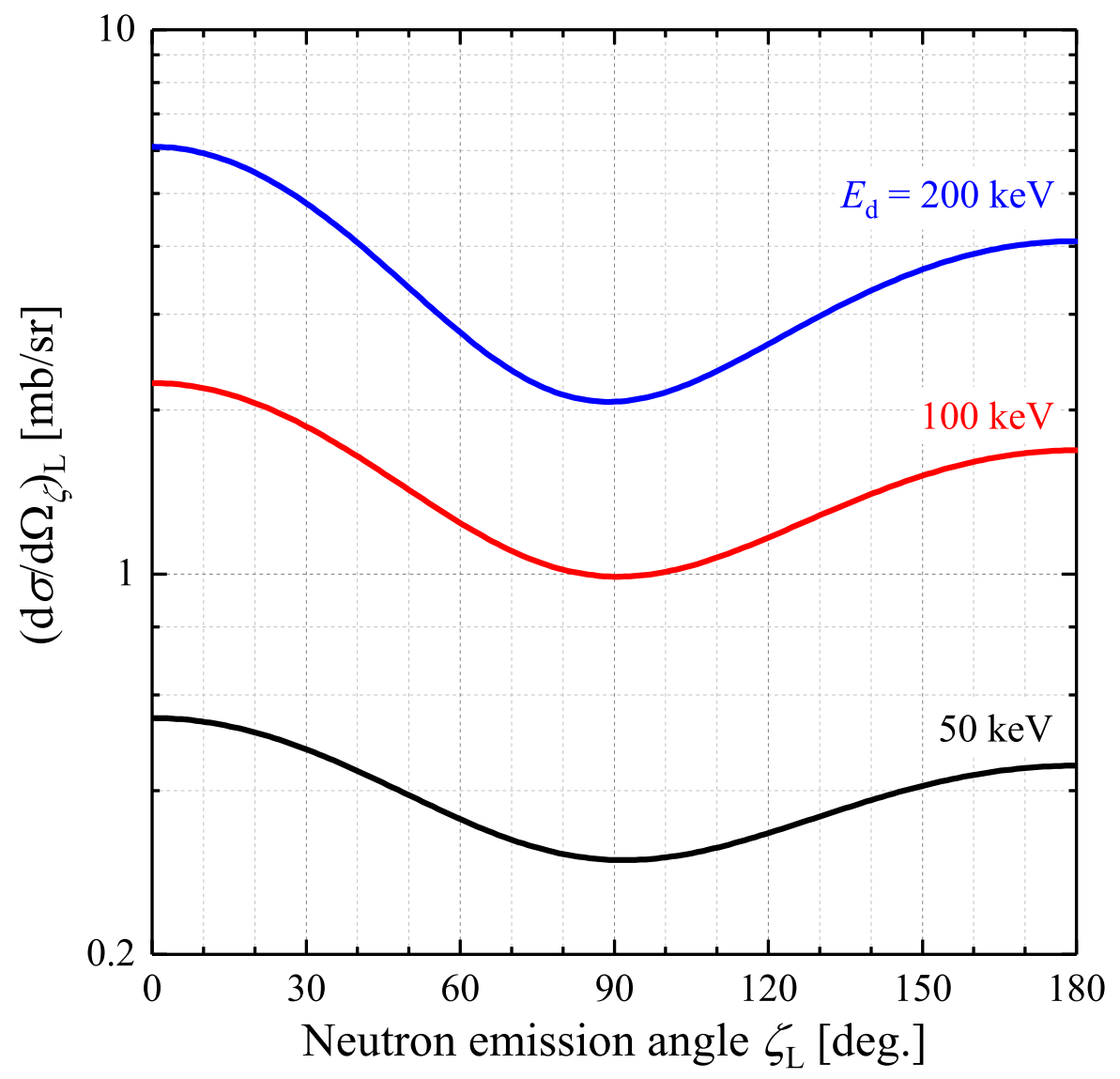

Figure 4. Differential cross-section of the $\mathrm{D}(\mathrm{d}, \mathrm{n})^{3} \mathrm{He}$ reaction in the laboratory system for deuteron energy $E_{\mathrm{d}}=50,100$ and $200 \mathrm{keV}$. 

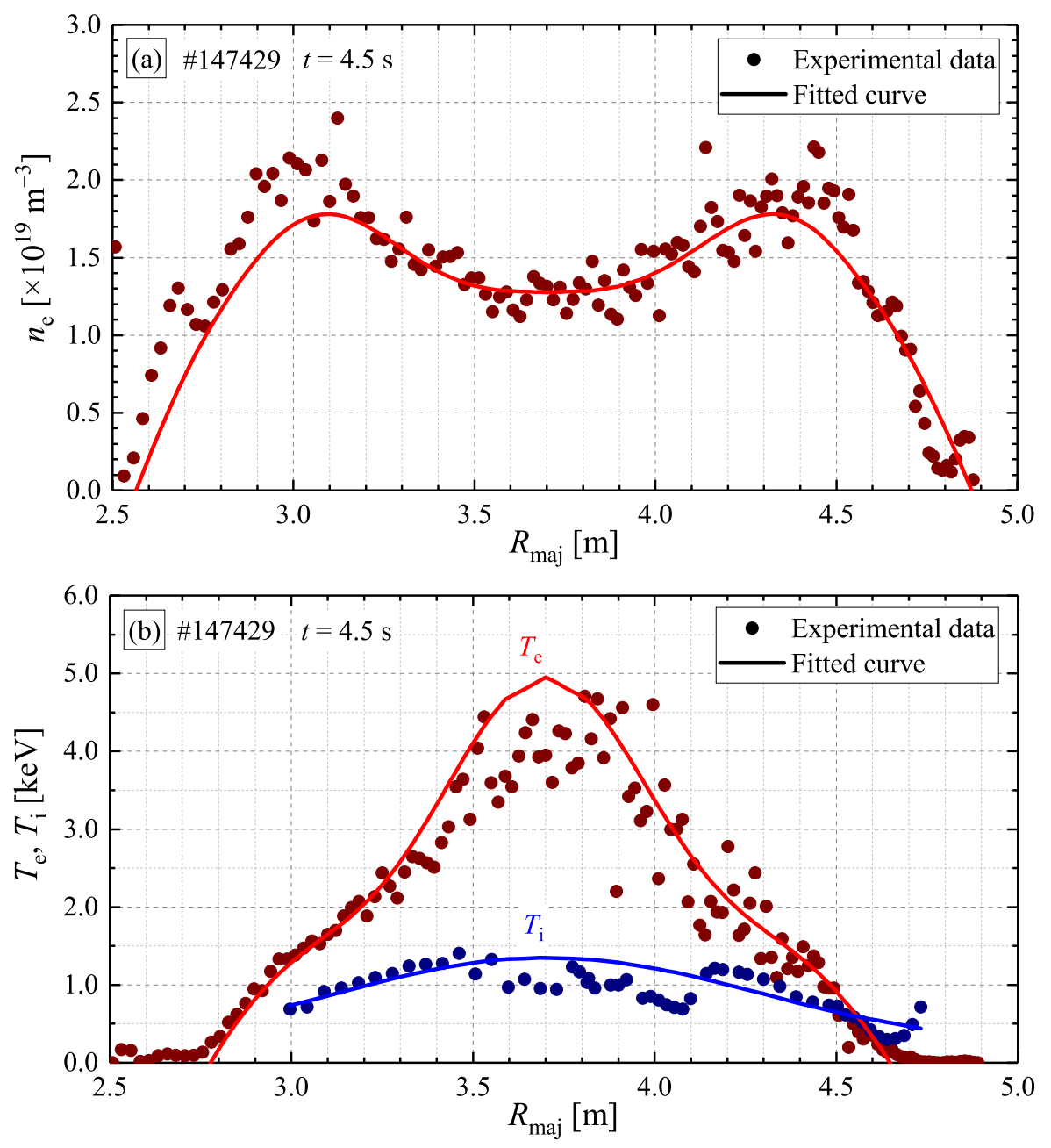

Figure 5. Profiles of (a) electron density and (b) electron and ion temperatures measured in shot \#147429 and fitted for numerical analysis. 

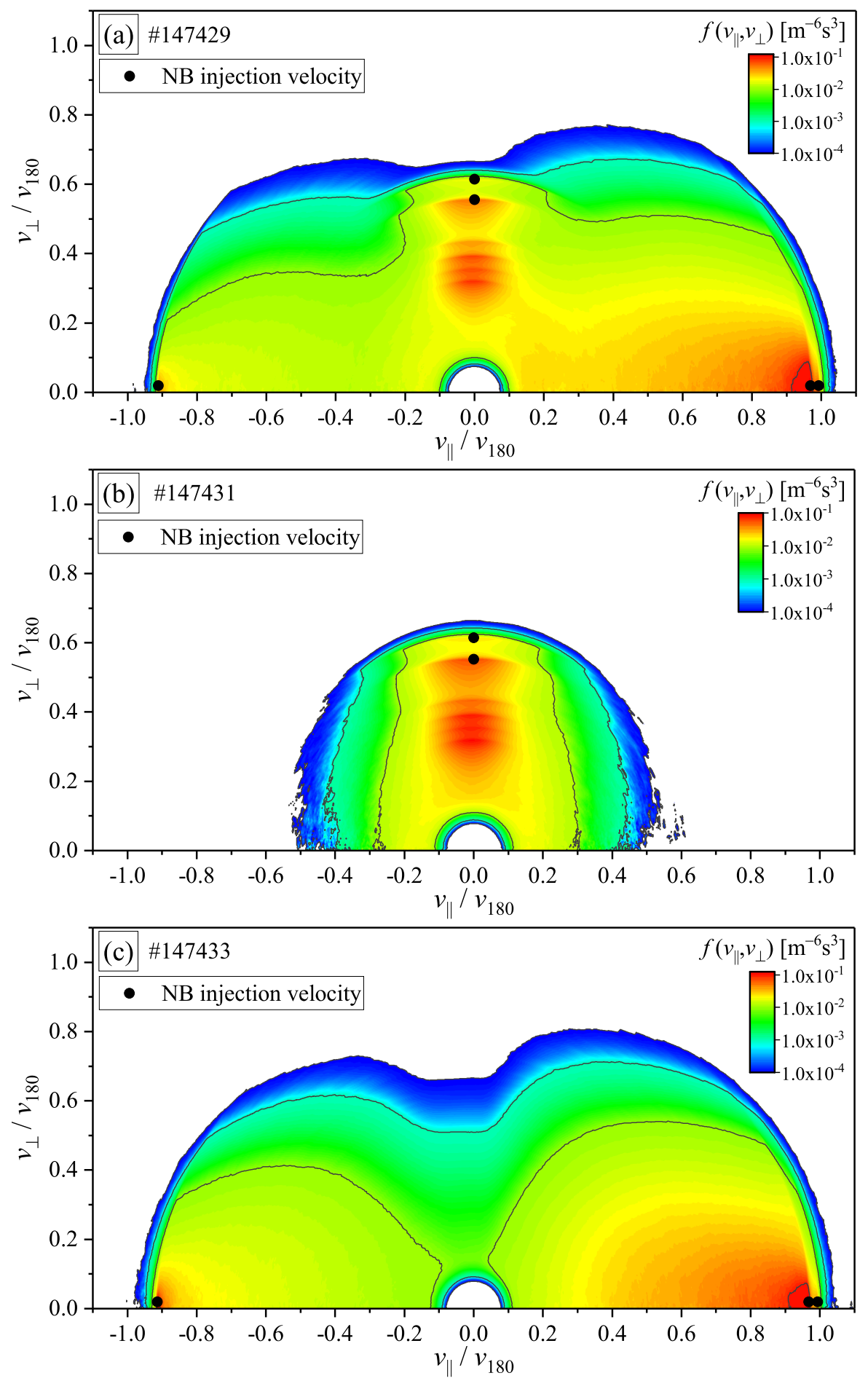

Figure 6. Volume-averaged energetic-deuteron velocity distribution functions obtained by numerical analyses for the cases of (a) tangential and perpendicular NB injection (\#147429), (b) perpendicular injection (\#147431) and (c) tangential injection (\#147433). $v_{180}$ is the $180-\mathrm{keV}$ deuteron speed. 

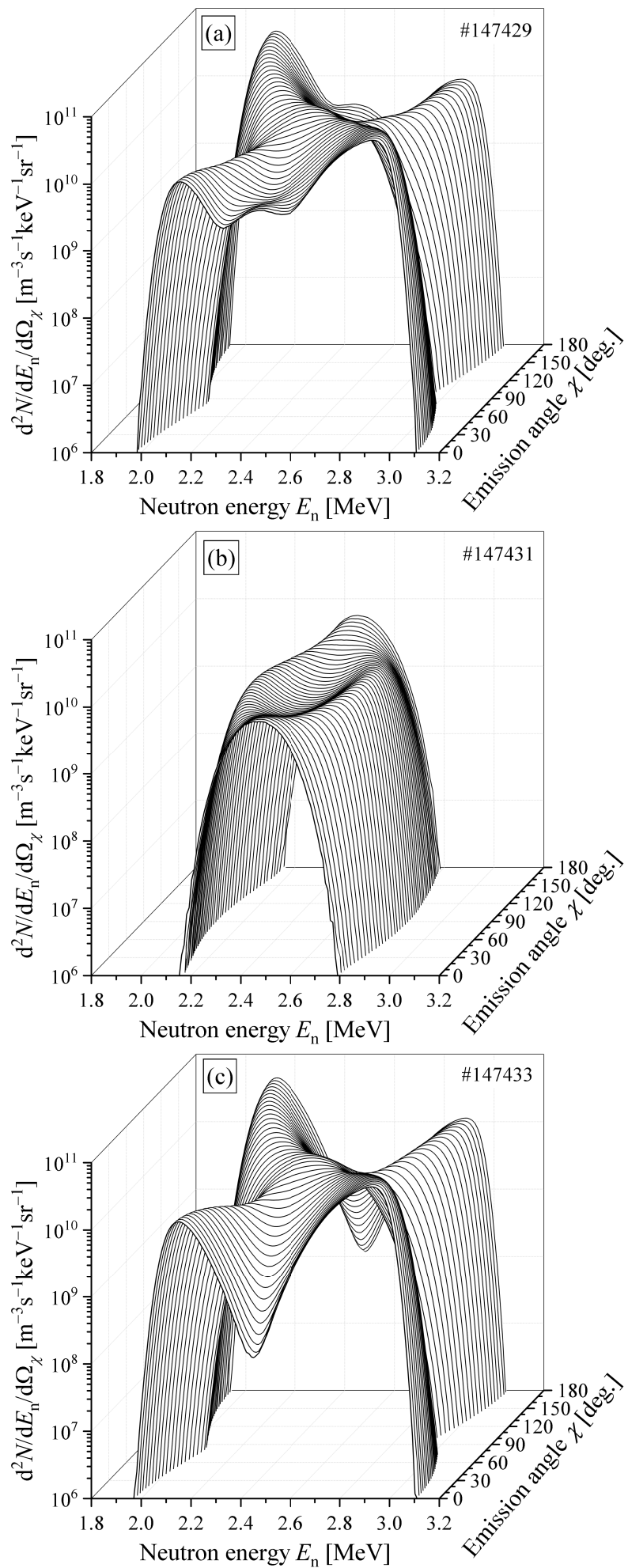

Figure 7. Volume-averaged double-differential neutron emission spectra obtained from numerical analyses for (a) tangential and perpendicular NB injection (\#147429), (b) perpendicular injection (\#147431) and (c) tangential injection (\#147433). 


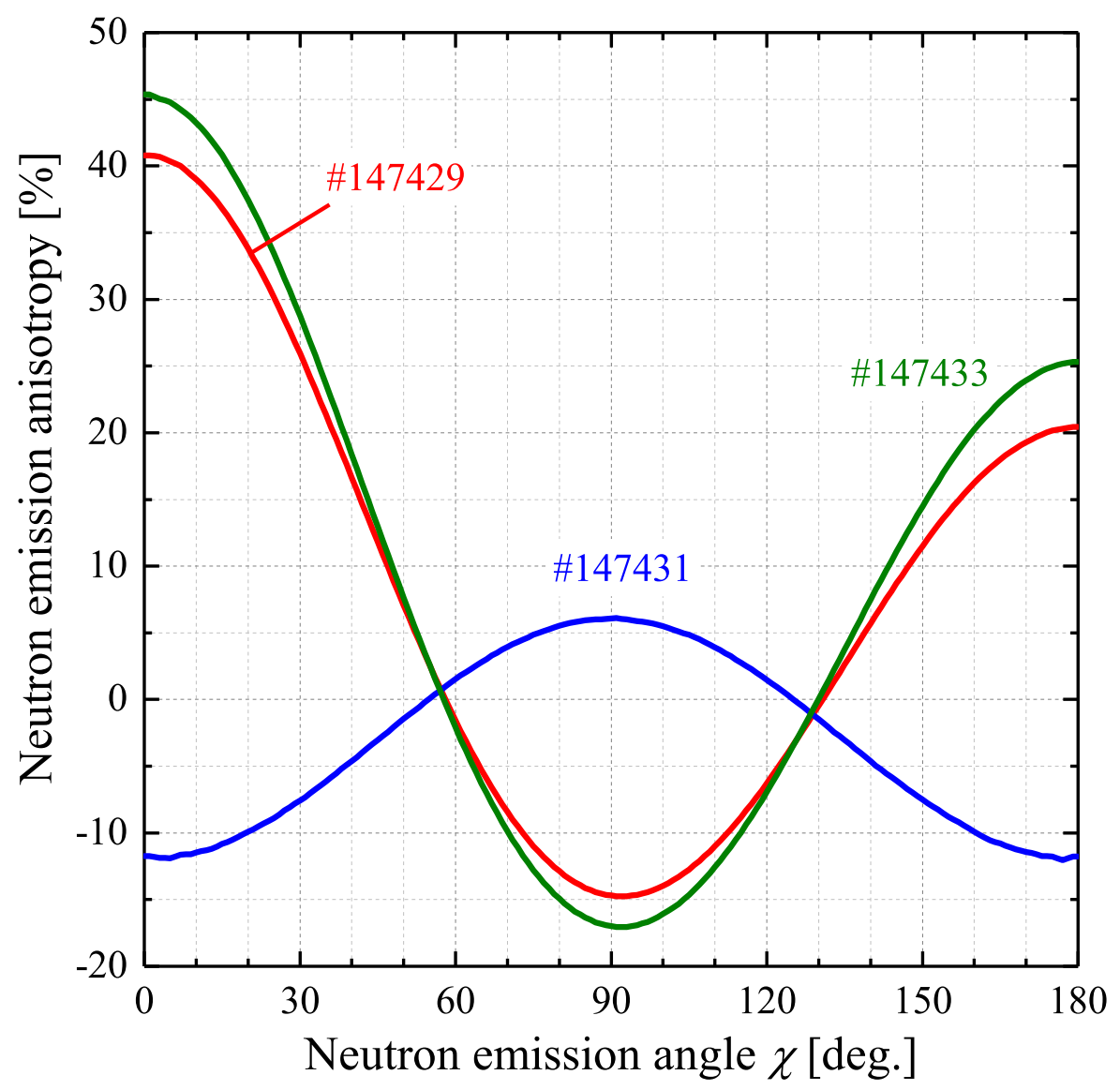

Figure 8. Neutron emission anisotropy defined as the relative difference between calculated angular distribution of neutron spectra and the angular distribution for isotropic emission. 